\title{
Histochemical Localization of Enzymes in the Embryonic Chick Liver'
}

\author{
JAMES L. CONKLIN \\ Department of Anatomy, The University of Michigan, Ann Arbor, Michigan
}

\begin{abstract}
Oxidative and hydrolytic enzymes were localized histochemically in the developing chick liver and in liver grown as chorioallantoic (CAM) transplants or as explants in organ culture.

In the in situ liver, parenchymal cells exhibited an increase in succinate (SDH) and a glycerophosphate (GDH) dehydrogenase activity and a decrease in NADP diaphorase (TPND) during development. NAD diaphorase (DPND) and nonspecific esterase (NSE) activity remained constantly high throughout the period studied. Within the stromal cells, DPND and NSE activity decreased and SDH, GDH and alkaline phosphatase (ALP) increased. The ALP activity of the sinusoidal cells appeared to increase coincident with a redistribution of TPND within the cytoplasm of the parenchymal cells.

In CAM transplants, the enzyme activity of all tissues was nearly the same as the five day liver even though the total age of the tissues was 15 days.

Liver explants exhibited enzyme activity similar in part to both the 5 and 15 day liver.

In both explants and transplants, ALP activity was highest in areas where the parenchymal-sinusoidal relationship was maintained and absent in areas where the parenchyma and sinusoidal cells were not in contact.
\end{abstract}

In a recent report (Conklin, '64), the cytochemical localization of lipid, glycogen and the enzyme, lactate dehydrogenase $(\mathrm{LDH})$, in the chick hepatic epithelium was described. It was noted that a correlation existed between the glycogen and lipid content of the hepatic cells and the ontogenesis of certain of the $\mathrm{LDH}$ isozymes (Nebel and Conklin, '64). Also noted was an alteration in $\mathrm{LDH}$ isozymes of liver which had been transplanted to the chorioallantoic membrane (CAM) of the chick.

The study of the embryonic liver has been extended to include additional enzymes, both oxidative and hydrolytic, in order to further characterize the developing hepatic parenchyma. In addition, a comparison has been made between enzyme distribution in epithelial and connective tissue cells.

Finally, since preliminary observations indicated a change in the LDH activity of liver transplants, enzyme activity was also studied in liver grown both in vitro, as organ explants, and in vivo, as chorioallantoic transplants.

\section{MATERIALS AND METHODS}

The material consisted of excised samples of liver from 20 five day and 5515 day (Hamburger-Hamilton, '51) white leghorn chick embryos and livers grown as transplants (45) and explants (20). In removing samples for enzyme demonstration or transplantation samples were taken at random from the liver. Since samples of the same age or subjected to the same treatment exhibited similar enzyme activity it is probable that regional variation within the liver was not a significant factor in the results obtained.

For transplantation livers were taken from five day donor embryos and grown for ten days in eight day host embryos. The liver of the five day embryo was removed aseptically and placed in sterile, avian saline. It was then cut into small pieces, 1-2 $\mathrm{mm}$ in size, which were used as the transplant. Preparation of the host embryo consisted of cutting a $3 \times 5 \mathrm{~mm}$ rectangle in the egg shell with a dental saw, removing the shell membrane and gently (with a sterile needle) breaking a small vessel in the chorioallantoic membrane (CAM) so that a drop or two of blood leaked onto the surface of the CAM. When leakage was judged to be excessive

1 Research supported by grant HD 00557 from NICHD, National Institutes of Health and grant ACS IN 40-D from the University of Michigan Cance Research Institute. 
the egg was not utilized for transplantation. The excised liver sample was placed directly on the break in the vessel with sterile forceps and the shell hole sealed with sterile scotch tape. Once sealed the eggs were not reopened until the tissue sample was removed although tissues were visually examined (through the tape) for viability during the incubation period.

In culturing, liver samples were excised as for transplanting, placed on rafts of stainless steel mesh and floated on nutrient medium (Trowell's T8) in plastic organ culture dishes. The cultures were maintained at $35 \mathrm{C}$ and gassed continuously with $95 \%$ oxygen and $5 \%$ carbon dioxide. Cultures were maintained for nine days and the medium was changed every three days.

In preparation for enzyme demonstration, liver samples were placed on dry ice until frozen, then mounted on a microtome chuck and sectioned at $2 \mu$ in a cryostat at $-20 \mathrm{C}$. The sections were mounted on coverslips, allowed to thaw, immersed in acetone at $4 \mathrm{C}$ for 15 seconds to extract neutral fat droplets, and placed in the substrate mixture.

To demonstrate a glycerophosphate dehydrogenase (GDH) the substrate mixture contained in final concentration, sodiumDL- $\alpha$ glycerophosphate, $0.02 M$; oxidized nicotinamide adeninedinucleotide (NAD), $0.3 \mathrm{mg} / \mathrm{ml}$; Nitro BT, $0.5 \mathrm{mg} / \mathrm{ml}$; tris (hydroxymethyl) aminomethane (Tris) buffer, $0.12 \mathrm{M}, \mathrm{pH} 7.0$, and phenazine methosulfate (PMS), $0.5 \mu \mathrm{g} / \mathrm{ml}$. For the demonstration of succinate dehydrogenase (SDH), the incubation mixture contained sodium succinate, $0.07 M$, Nitro BT, Tris buffer and PMS. Diaphorases (DPND and TPND) were demonstrated by incubating sections in a solution containing phosphate buffer, $0.12 M, \mathrm{pH} 7.2$; Nitro $\mathrm{BT}$ and reduced $\mathrm{NAD}\left(\mathrm{NADH}_{2}\right)$ or $\mathrm{NADPH}_{2}, 0.5 \mathrm{mg} / \mathrm{ml}$, respectively (Conklin et al., '62). Alkaline phosphatase (ALP) and nonspecific esterase (NSE) activity was demonstrated by the method of Burstone ('62) employing naphthol-AS phosphate and a naphthyl butyrate as substrates, respectively.

The standard incubation procedure for diaphorase and phosphatase was $15 \mathrm{~min}$ utes at $37 \mathrm{C}$ while 30 minutes incubation at $37 \mathrm{C}$ was utilized for all other methods.
The chemicals employed in the substrate mixture were obtained from Sigma Chemical Co. with the exception of NAD, $\mathrm{NADH}_{2}$ and $\mathrm{NADPH}_{2}$ (Calbiochem) and Nitro BT (Dajac Laboratories).

After incubation, sections were rinsed in distilled water followed by $10 \%$ neutral formalin, mounted in glycerogel and examined and photographed as soon as possible after mounting.

Additives were combined as needed with certain of the incubation mixtures as follows: coenzyme $Q$ (ubiquinone 30 ), nicotinamide, sodium amobarbital, sodium azide and potassium cyanide. The effect of these additives on the demonstration of enzyme activity are discussed in another report (Conklin, '66).

\section{OBSERVATIONS}

The five day chick liver is composed of branching cords of epithelial cells which are separated from each other by prominent areas of mesenchyme (figs. 1, 23). Within the mesenchyme in an apparently unorganized arrangement are numerous blood vessels. Small, blood sinusoids which are present are surrounded by mesenchyme and not in close contact with the parenchymal cells. Enzyme distribution in the five day liver was evaluated by comparing the enzyme activity of the parenchyma, stroma and lining cells of the venous channels.

By the fifteenth day of development, the architecture of the liver is apparent. As illustrated in figures 3 and 4 , the organization of the chick liver is similar to that reported for other species (Elias, '49). Within the connective tissue of the portal area are lobular branches of the bile duct, hepatic artery, and portal vein. Numerous branching cords of epithelial cells radiate from the bile ducts. Between the epithelial cords, the mesenchyme is no longer prominent and most of the interepithelial space is filled by the sinusoids and their lining cells. The sinusoids, which originate as tributaries from the lobular veins, extend from the portal area to the central veins in an interanastomosing pattern. The central veins, in turn, empty into larger, collecting veins which are surrounded by connective tissue. 
The lobular pattern of the chick liver is poorly defined and both blood sinusoids and parenchymal cell cords probably interanatamose with like structures in adjacent lobules. In evaluating enzyme distribution, the enzyme activity of the parenchymal cells was contrasted with the activity of cells in the connective tissue and with the lining cells of the veins and sinusoids.

\section{Normal development}

In the five day liver (tables 1 and 2 ) (figs. 1, 2, 7, 13, 14, 19, 23), the parenchymal cells displayed strong TPND, DPND and NSE activity while SDH activity was weak. Only slight GDH activity was present and ALP activity was absent. Of these enzymes, SDH and DPND (fig. 14) appeared to be localized within the mitochondria (particulate dye particles) while TPND (fig. 2) and NSE were extramitochondrial in localization (diffuse dye particles). GDH activity was too weak to be localized intracellularly.

Within the stromal cells, all enzymes were weakly active with the exception of GDH and ALP which were negative. SDH was weakly active in the sinusoidal lining cells, GDH activity was absent while TPND, DPND, NSE, and ALP activity occurred in trace amounts. Intracellular localization of these enzymes within the stromal or sinusoidal cells was not attempted because of the difficulty in resolving individual dye particles in the small cells.

In the 15 day liver (figs. 3-4, 8-10, $15-16,20,22,24-25)$ the parenchymal cells exhibited strong SDH, DPND and NSE activity. Weak TPND and GDH activity was present while ALP activity was not demonstrable. SDH (fig. 20) and GDH (fig. 22) activity occurred only within the mitochondria. DPND activity (fig. 16) was found both intra- and extramitochondrially while TPND (fig. 5) and NSE activity (fig. 25) was nonmitochondrial in localization.

The stromal cells displayed strong SDH activity and weak TPND activity. DPND activity was absent while trace activity of GDH, NSE and ALP was observed. Strong SDH and ALP activity (figs. 9-10) was present in the sinusoidal cells while all other enzymes occurred in trace amounts.

In summary, it was observed that between the fifth and fifteenth day of development the parenchymal cells displayed an increase in SDH and GDH activity and a decrease in TPND activity. There was no apparent change in the activity of DPND, NSE and ALP, the latter being negative throughout the period studied. Within the stromal cells, DPND and NSE activity declined, while the activity of SDH, GDH and ALP increased. No change in TPND activity was observed. Within the sinusoids, TPND, DPND and NSE activity remained unchanged while SDH, GDH and particularly ALP activity increased.

Within the hepatic lobule, TPND activity (fig. 4) was most intense in the cells which were adjacent to connective tissue. In the parenchymal cells adjacent to the sinusoids, TPND activity in the five day liver was uniformly distributed throughout the cytoplasm. In the 15 day liver, all activity was confined to the cytoplasm adjacent to the sinusoid (fig. 5). All other enzymes were uniformly distributed throughout the cytoplasm of the parenchymal cells.

ALP activity which was intense only in the vascular lining cells increased in activity in the sinusoids in a central to peripheral distribution (fig. 8). Only weak activity was present in the lining cells of the intralobular veins while the cells of the distal portion of the sinusoids and the lining cells of the collecting veins exhibited intense ALP activity. None of the other enzymes studied exhibited variations in activity as a consequence of intralobular distribution.

\section{Chorioallantoic transplants}

When samples of liver from five day embryos were transplanted to the CAM of seven day chick embryos alterations in the activity of certain of the enzymes were noted (figs. 6, 11-12, 18, 26). In the CAM transplants, only DPND activity was strong while TPND, SDH and NSE activity was weak. GDH and ALP activity could not be demonstrated. Within the parenchymal cells, TPND (fig. 6) and DPND (fig. 18) exhibited a particulate 
pattern of activity suggesting that the enzymes were localized within the mitochondria. SDH appeared to be located both intra- and extramitochondrially, while NSE activity (fig. 26) was distributed extramitochondrially. All enzymes were distributed throughout the cytoplasm and no polarization of activity was observed.

Within the stromal cells, only weak or trace activity of TPND, DPND, SDH and NSE was observed, while GDH and ALP activity was absent. The same pattern of activity was observed in the sinusoidal lining cells with the exception of ALP. In those areas where the sinusoidal lining cells were in close proximity to the parenchymal epithelium, the sinusoidal cells exhibited strong ALP activity (fig. 11). In other areas of the transplant, where reorganization had occurred in the form of an cnlargement of the sinusoid and a dissociation of the sinusoidal and parenchymal cells, only weak ALP activity was observed (fig. 12). Specific alterations in the intralobular distribution of enzymes was not seen.

When development of the CAM transplant was contrasted with the normal development of the liver the following differences were noted. Although the total age of the liver transplant was 15 days, the parenchymal cells exhibited the same intensity and distribution of enzyme activity as did the five day liver. The same was true of the stromal cells and sinusoidal lining cells except for ALP activity. In sinusoids which were similar in size and arrangement to those of the 15 day liver, the intensity of ALP activity was similar to that of the sinusoidal cells of the 15 day liver.

\section{Organ explants}

The parenchymal cells in the liver explants (figs. 19, 21) exhibited strong DPND and NSE activity and weak or trace TPND, SDH and GDH activity. ALP activity was absent. $\mathrm{GDH}$ and $\mathrm{SDH}$ activity was similar to that of the five day liver while TPND and DPND activity was like that of the 15 day liver. Within the cells DPND activity occurred both intraand extramitochondrially and TPND, NSE and SDH appeared to be localized outside of the mitochondria. TPND activity, where present, was more intense on the sinusoidal perimeter of the cell as in the normal liver.

The stromal cells displayed strong DPND activity while other enzymes were weakly active (TPND, SDH, NSE) or absent (GDH, ALP). The strong DPND activity was in contrast to the weak activity present in normal or transplanted liver. The activity of other enzymes was similar to that seen in the five day liver. Within the sinusoidal lining cells, enzyme activity was similar to that of the five day liver except, again, for ALP. ALP activity was similar to that of the CAM transplants in that it was intense in areas where sinusoids and parenchyma were in association and decreased or absent where this association was altered.

\section{DISCUSSION}

Previous studics of the developing hepatic parenchyma have demonstrated a progressive increase in metabolic products such as lipid, glycogen and bile pigments (Dalton, '37; Ballard and Oliver, '63; Conklin, '64). Not unexpectedly, the activity of certain enzymes as demonstrated both biochemically and histochemically has been shown to increase concomitantly Solomon, '59; Ballard and Oliver, '63; Conklin, '64; and Strittmatter, '65).

One implication of these results is that much of the developmental process is devoted to the acquisition of function as reflected by the increase in enzyme activity and storage of inclusions. This conclusion is undoubtedly an oversimplification and, in reality, cellular maturation is a multifold process, the two extremes of which are the acquisition of certain functions at the expense of others. Perhaps more significant are the subtle shifts in function that occur and are difficult to measure by most methods since they do not involve an all or none response.

It might be expected that in the formation of an organ different tissues would initially possess common properties but would acquire different properties during organogenesis. The properties at the conclusion of organogenesis would then be those of the organ and might be different from the original properties exhibited by 
the individual tissues. It seemed of interest then to examine the changes in tissue characteristics which occur during organ formation. Also of interest was the examination of tissue properties of a transplanted organ to determine if alteration of organ formation is accompanied by a change in tissue enzymes.

In the present study, the enzymatic activity of different tissues of the developing liver have been examined during a period of extensive change, i.e., 5 to 15 days. The selection of this period does not imply that tissue properties do not change during the remainder of embryogenesis since the growth of a tissue is a continuing process. However, subsequent changes may be more subtle and less demonstrable by the methods employed.

As indicated in table 1 , most of the differences in enzyme activity studied in the normal liver were quantitative. In the five day liver, TPND, DPND, SDH, and NSE were present in both epithelial and connective tissue cells. GDH which was present only in the hepatic parenchyma and ALP which occurred only in the sinusoidal cells were the only enzymes which were not demonstrable in all tissues. In the 15 day liver, all enzymes were present in all tissues with the exception of DPND which was absent from connective tissue and ALP which did not appear in the parenchymal cells. Loss of enzyme activity was exemplified by the decrease in TPND activity of the parenchyma and the reduction of DPND and NSE activity in the connective tissue. An increase in enzyme activity was exhibited by the elevation of ALP activity in the sinusoids and an increase in SDH and GDH in all tissues. The increase in SDH activity in all tissues suggests a common increase in non NAD-linked oxidative metabolism with a marked decrease in NADlinked dehydrogenase activity in connective tissue cells as indicated by the decline in DPND activity. The elevation of ALP activity in sinusoidal lining cells may be related to the specialization of these cells for purposes of transport. The decrease in NSE activity in the connective tissue cells and the decrease in parenchymal TPND activity are not easily evaluated. The latter enzyme might be expected to increase in activity in view of the increase in lipid within the parenchymal cells (Conklin, '64).

Even more interesting than the shifts in total enzyme activity in different tissues was the alteration in intracellular localization of enzymes which was observed in the parenchymal cells. As indicated in table 2 , during normal development certain enzymes were associated with certain cellular organelles. Some enzymes were confined to the mitochondria ( $\mathrm{SDH}, \mathrm{GDH}$ ), some had an extramitochondrial localization (TPND, NSE) and one was found in both sites (DPND).

TABLE 1

Histological localization of enzyme activity

\begin{tabular}{|c|c|c|c|c|c|c|}
\hline \multirow{2}{*}{ Enzyme } & \multicolumn{3}{|c|}{ 5-Day } & \multicolumn{3}{|c|}{ 15-Day } \\
\hline & Parenchyma & Stroma & Sinusoids & Parenchyma & Stroma & Sinusoids \\
\hline TPND & strong & weak & trace & weak & weak & trace \\
\hline DPND & strong & weak & trace & strong & 0 & trace \\
\hline SDH & weak & weak & weak & strong & strong & strong \\
\hline GDH & trace & 0 & 0 & weak & trace & trace \\
\hline NSE & strong & weak & trace & strong & trace & trace \\
\hline ALP & 0 & $\mathbf{0}$ & trace & 0 & trace & strong \\
\hline TPND & trace & $\begin{array}{c}\text { Explant } \\
\text { weak }\end{array}$ & trace & \multicolumn{3}{|c|}{ CAM transplant } \\
\hline DPND & strong & strong & trace & strong & trace & trace \\
\hline SDH & trace & weak & trace & weak & weak & trace \\
\hline GDH & trace & 0 & 0 & trace & 0 & 0 \\
\hline NSE & strong & trace & trace & strong & trace & trace \\
\hline ALP & 0 & 0 & strong-0 & 0 & 0 & strong-trace \\
\hline
\end{tabular}

Enzyme activity was visually determined on the basis of the intensity of staining and arbitrarily indicated as strong, weak, trace or negative $(0)$. 
TABLE 2

Intracellular localization of enzyme activity

\begin{tabular}{|c|c|c|c|c|}
\hline Enzyme & $\begin{array}{l}\text { 5-Day } \\
\text { liver }\end{array}$ & $\begin{array}{l}\text { 15-Day } \\
\text { liver }\end{array}$ & $\begin{array}{l}\text { Liver } \\
\text { explant }\end{array}$ & $\underset{\text { transplant }}{\text { Cam }}$ \\
\hline TPND & $\mathrm{E}$ & $\mathrm{E}$ & $\mathbf{E}$ & M? \\
\hline DPND & $\mathrm{M}$ & $\mathrm{M}, \mathrm{E}$ & $\mathrm{M}, \mathrm{E}$ & M \\
\hline $\mathrm{SDH}$ & $\mathrm{M}$ & $\mathrm{M}$ & $\mathrm{E}$ & $\mathbf{M}, \mathbf{E}$ \\
\hline GDH & - & $\mathbf{M}$ & - & - \\
\hline NSE & $\mathbf{E}$ & $\mathrm{E}$ & $\mathbf{E}$ & $\mathbf{E}$ \\
\hline ALP & 一 & - & - & - \\
\hline
\end{tabular}

Enzyme activity was localized as either mitochondrial ( $M$ ), or extramitochondrial ( $E$ ). - indicates the absence of demonstrable activity.

The localization of the latter was similar to that previously reported for lactate dehydrogenase (Conklin, '64). Although the organellar localization of TPND was not determined this enzyme was most interesting in that during development it became restricted to a particular area of the cytoplasm, i.e., adjacent to the sinusoids. Perhaps, this distribution was in response to the activity of the lining cells of the sinusoid and also the fact that it is through this area of the cell that materials exit and enter from the vascular system. This change in localization may also account for the reduction in total activity which was observed. A similar polarization of enzyme activity in parenchymal cells was noted by Novikoff and Essner ('60) who reported ATPase activity adjacent to the bile canaliculus. The fact that TPND activity was consistently higher in the cytoplasm of cells adjacent to connective tissue (fig. 4) suggests some kind of causal relationship between the tissue arrangement. Although the connective tissue cells contain abundant mitochondrial LDH activity (Conklin, '65) they are lacking in DPND activity. Precise variation in the intralobular distribution of function has been noted in mammalian liver (Novikoff and Essner, '60). This was not observed in the embryonic chick liver except for a slight increase in sinusoidal ALP activity in the periphery as compared with the center of the lobules.

Transplantation of liver resulted in certain notable alterations in development as indicated by enzyme activity. With the exception of ALP, the enzyme activity of liver-CAM transplants was similar to that of the five day liver even though the total age of the transplant was 15 days.
As the tissue appeared viable, it would seem that the effect of transplantation to the CAM was to alter normal enzymogenesis.

Determination of enzyme activity in a histochemical system is interpreted from the variation in the reduction of a dye. Therefore, an increase in dye reduction in a given period of time is either the result of an increase in the ability of the enzyme to act upon the substrate (activation), due to an increase in the amount of enzyme present (synthesis), or both. While the results indicate an alteration in activation and/or synthesis of enzymes in the transplanted tissues it is not possible to conclude from the present data which is of the greater significance. Also, the causative factor or factors which result in the altered activity or synthesis of enzymes in the transplanted liver cannot be identified. While there are numerous possibilities such things as differences in in situ gaseous environment (Moscona, '59) or even total tissue mass (Trinkaus, '56) are not to be excluded from consideration. One possible way of examining the importance of local environment would be to compare the result of coelomic and CAM transplantation

Liver grown in vitro exhibited enzyme activity intermediate between that of the 5 and 15 day liver. Again, the factors responsible for the altered pattern of activity are, at present, too numerous to consider.

A consistent finding in both CAM transplants and cultured explants was an increase in ALP activity in the sinusoidal lining cells when the cells were maintained in proximity to the parenchyma. This observation suggests some type of 
interaction between the lining cells and the parenchyma which results in the increased synthesis of ALP.

In conclusion, it has been illustrated that (1) in the early embryo different tissues of the liver are similar in both enzyme content and activity; (2) during liver formation the activity of some enzymes increase while others decline; (3) during organogenesis new enzymes appear in certain tissues; (4) the usual enzyme activity of an organ can be modified as the result of transplantation, and (5) there is a tendency for tissues to retain their initial, individual characteristics following transplantation rather than developing the properties which are characteristic of the total organ. Subsequent studies employing quantitative procedures may reveal more precisely the regulatory mechanisms which control enzymogenesis during liver formation.

\section{ACKNOWLEDGMENT}

I am indebted to Dr. Raymond H. Kahn for conducting the organ culture portion of this study. I would also like to acknowledge the technical assistance of Mrs. Lana Brock and Mrs. Constance Miller.

\section{LITERATURE CITED}

Ballard, F. J., and I. T. Oliver 1963 Glycogen metabolism in embryonic chick and neonatal rat liver. Biochem. Biophys. Acta, 71: 578588.

Burstone, M. S. 1962 Enzyme histochemistry and its application in the study of neoplasms. Academic Press, New York.

Conklin, J. L. 1964 A cytochemical study of lipid, glycogen and lactate dehydrogenase in the developing liver. J, Exp. Zool., 155: 151160 .

1965 Enzyme localization in the developing liver. Anat. Rec., 151: 447.

1966 Phenazine methosulfate; its use in evaluating activity of dehydrogenase systems of avian liver. Stain Technol., 41: 105-114

Conklin, J. L., M. M. Dewey and R. H. Kahn 1962 Cytochemical Iocalization of certain oxidative enzymes. Am. J. Anat., 110: 19-27.

Dalton, A. J. 1937 The functional differentiation of the hepatic cells of the chick embryo. Anat. Rec., 68: 393-410.

Elias, H. 1949 A re-examination of the structure of the mammalian liver. I. Parenchymal architecture. Am. J. Anat., 84: 311-334.

1949 A re-examination of the structure of the mammalian liver. II. The hepatic lobule and its relation to the vascular and biliary systems. Am. J. Anat., 85: 379 456.

Hamburger, V., and H. L. Hamilton 1951 A series of normal stages in the development of the chick embryo. J. Morph., 88: 49-92.

Moscona, A. 1959 Squamous metaplasia and keratinization of chorionic epithelium of the chick embryo in egg and in culture. Develop. Biol., 1: 1-23.

Nebel, E. J., and J. L. Conklin 1964 The development of lactic dehydrogenase isozymes in the chick embryo. Proc. Soc. Exp. Biol. and Med., 115: 532-536.

Novikoff, A. B, and E. Essner 1960 The liver cell, some new approaches to its study. Am. J. Med., 29: 102-110.

Solomon, J, B. 1959 Changes in the distribu. tion of glutamic, lactic, and malic dehydrogenases in liver cell fractions during development of the chick embryo, Develop. Biol., 1: 182198.

Strittmatter, C. F. 1965 Studies on avian xanthine dehydrogenases. Properties and patterns of appearance during development. J Biol. Chem., 240: 2557-2564.

Trinkaus, J. P. 1956 The differentiation of tissue cells. The American Naturalist, 90: $273-289$. 
PLATE 1

EXPLANATION OF FIGURES

All figures are of chick liver

1 TPND activity in five-day liver. Note the intense activity in the cords of epithelial cells (arrow) and the sparse activity in the connective tissue. $\times 400$.

2 TPND activity in five-day liver. Within the parenchymal cells (arrow), activity is uniformly distributed throughout the cytoplasm. $\times 1,500$.

3-4 TPND Activity in 15-day liver. Note the apparent reduction of activity in the parenchymal cells. Intense activity occurs in the cytoplasm of parenchymal cells adjacent to the periportal $(p)$ and sinusoidal ( $s$ ) connective tissue as well as that associated with the collecting veins $(c), \times 160, \times 400$. 

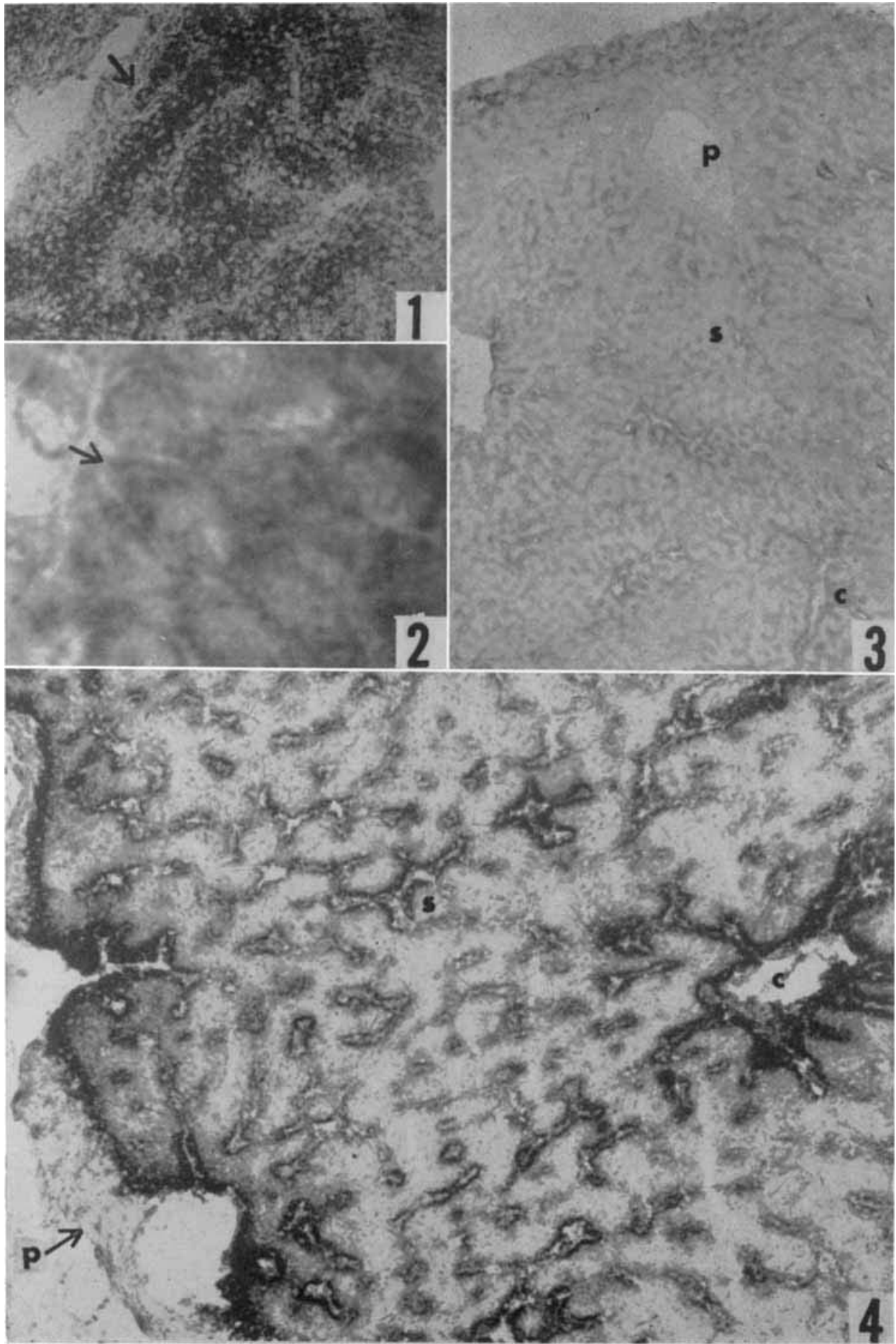


\section{PLATE 2}

EXPLANATION OF FIGURES

5 TPND activity in 15-day liver. The distribution of activity in relation to the sinusoid ( $s$ ) and bile canaliculus $(b)$ is clearly seen. $\times 1,500$.

6 TPND activity in parenchymal cells of a CAM liver transplant. Activity is uniformly distributed throughout the cytoplasm. Connective tissue cells (arrow) exhibit little activity. $\times 1,500$.

7 ALP activity in five-day liver. Weak activity is present in the lining cells of some of the sinusoids. $\times 400$.

8 ALP activity in 15-day liver. Weak activity is present in the lining cells of the lobular veins ( $i$ ) while strong activity occurs in cells lining the sinusoids and collecting veins $(c) . \times 160$. 

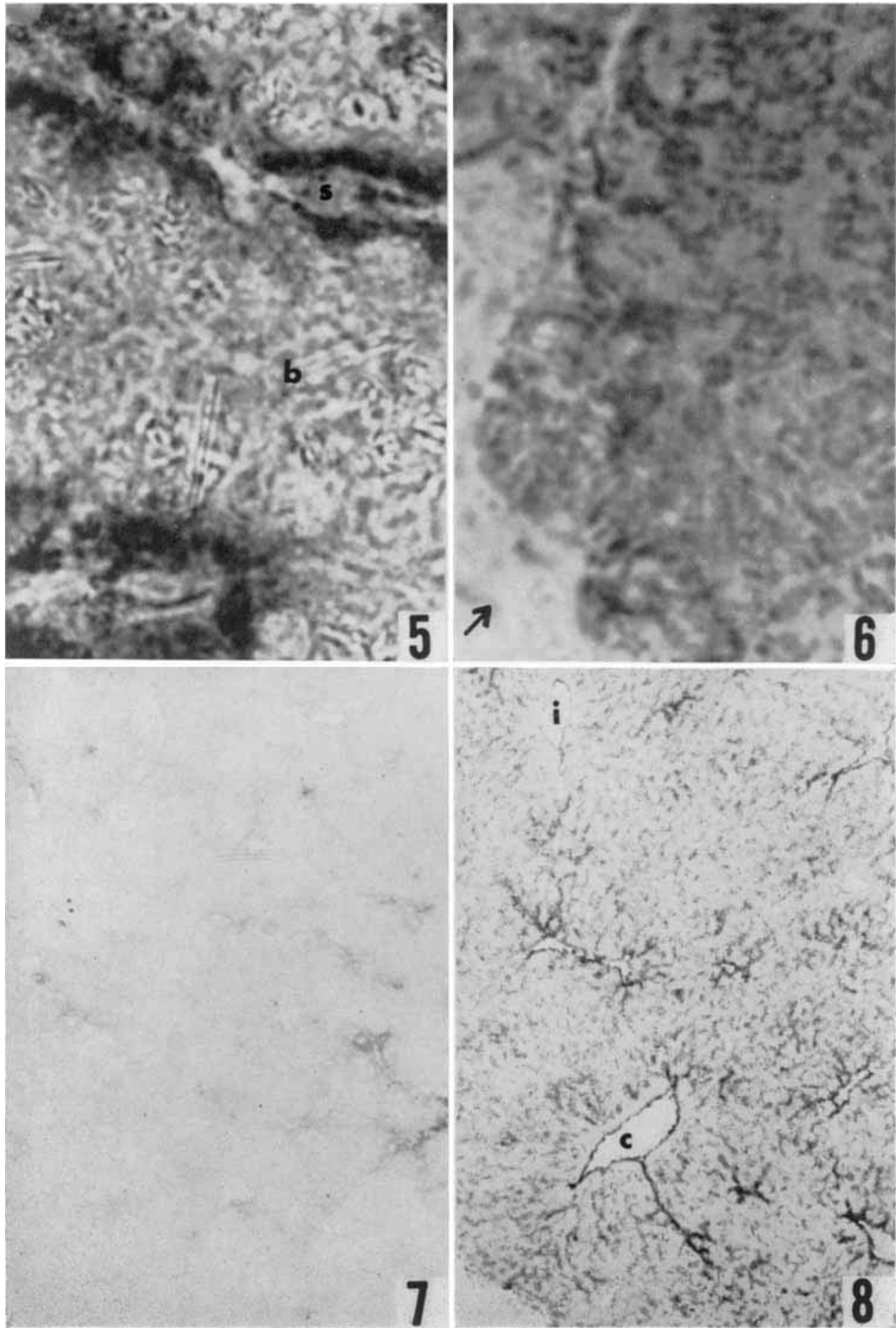
PLATE 3

EXPLANATION OF FIGURES

9-10 ALP activity in 15-day liver. The activity of the cells lining a collecting vein (fig. 9) and a sinusoid (fig. 10) is clearly seen. Weak activity may also occur in the connective tissue $(t)$ around the collecting vein (fig. 9). $\times 1,500$.

11-12 ALP activity in a CAM liver transplant. Where the integrity of the sinusoid ( $s$ ) has been maintained (fig. 11) activity is present (arrow) as in the normal liver while activity is absent from sinusoids in which the lining cells and parenchyma have become dissociated (fig. 12). $\times 1,500$. 

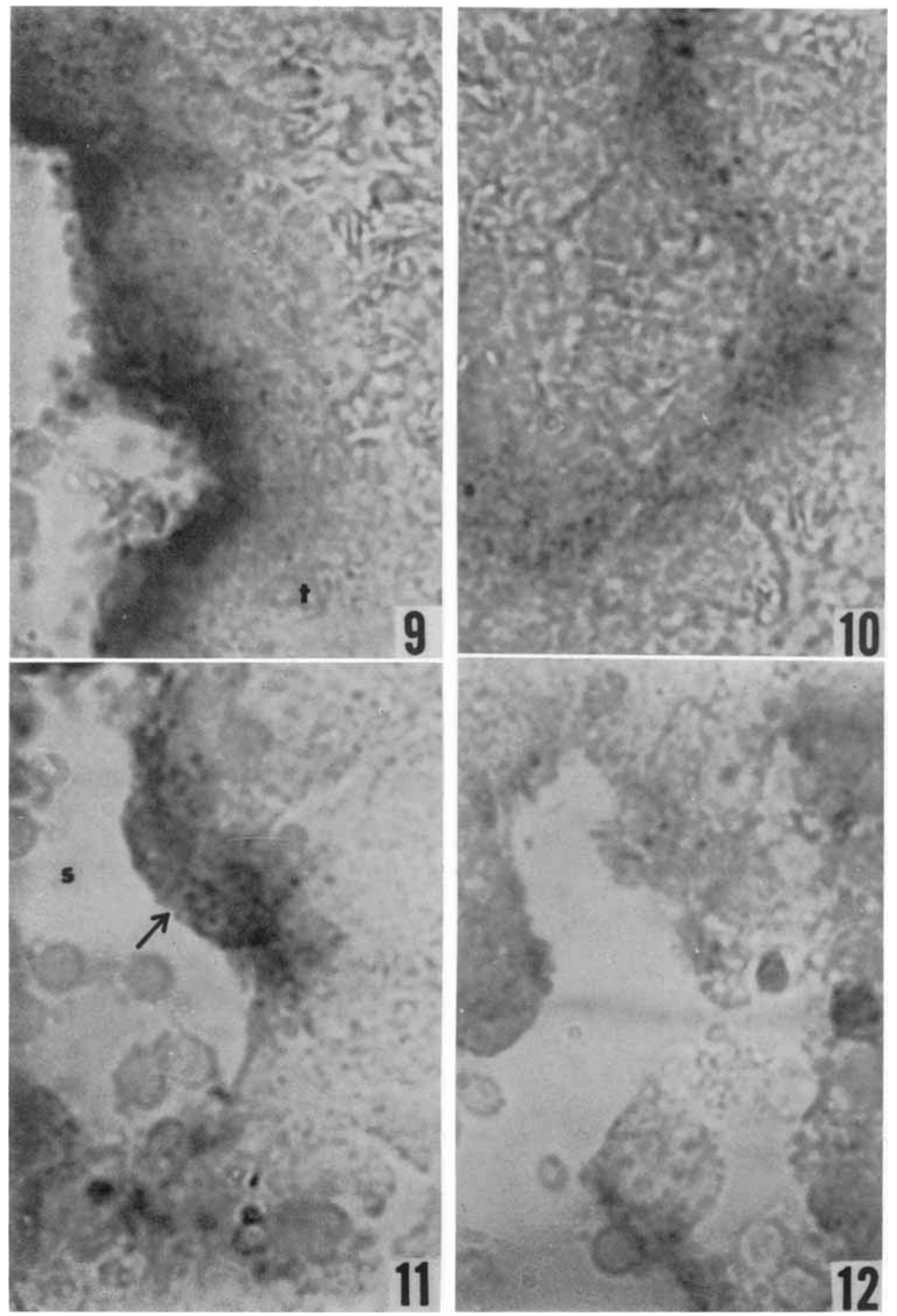
PLATE 4

EXPLANATION OF FIGUIEES

13-14 DPND activity in five-day liver. Note the intense activity in the cords of epithelial cells and weak activity in the connective tissue (arrow). Activity in the parenchymal cells (fig. 14) appears to be localized within the mitochondria. $\times 160, \times 1,600$.

15-16 DPND activity in 15-day liver. Strong activity occurs in the parenchyma while the vascular lining cells (arrow) are weakly positive. No activity occurs in the periportal connective tissue. Activity in the parenchymal cells (fig. 16) appears to be both intra- and extramitochondrial. $\times 160, \times 1,600$. 

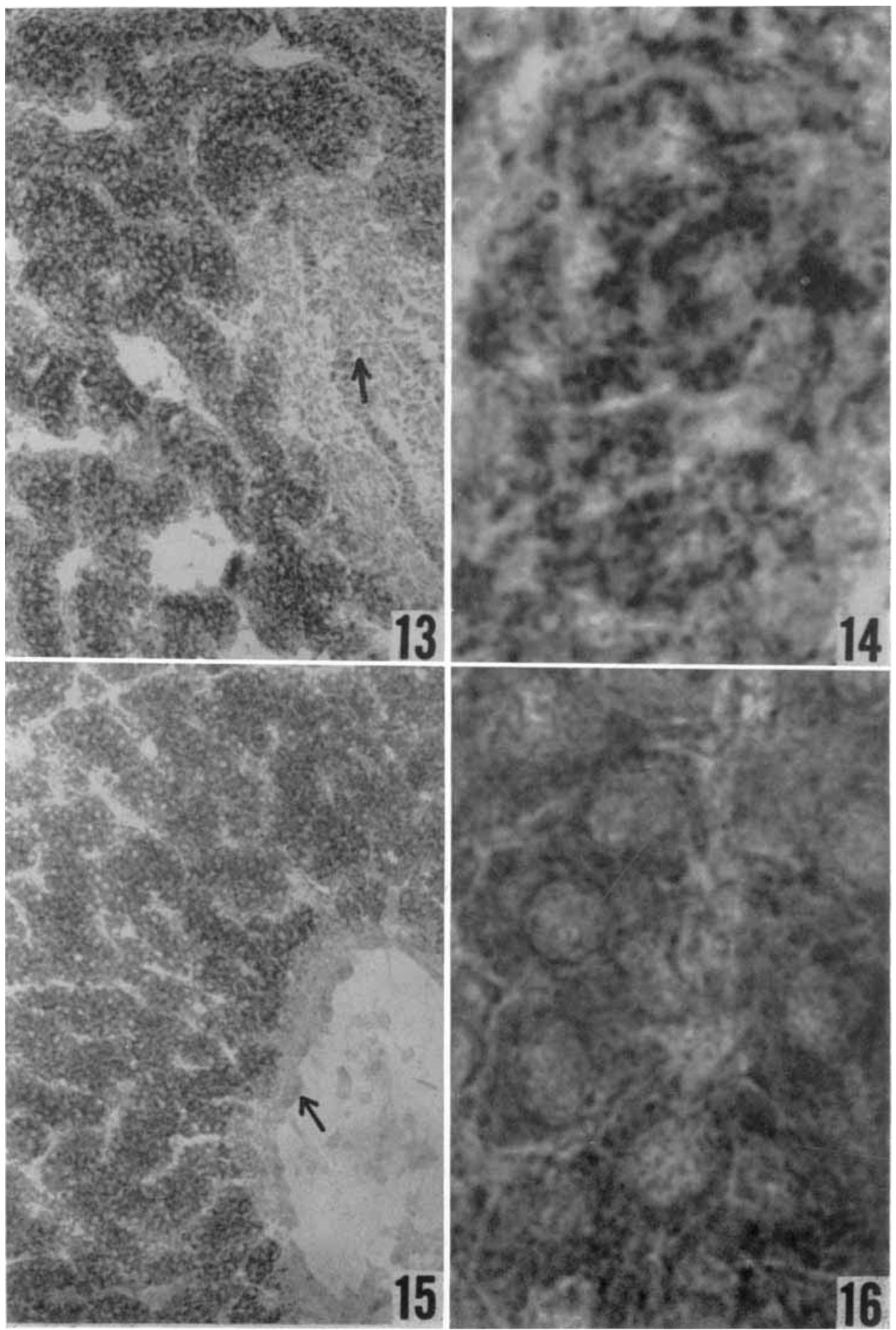
PLATE 5

EXPLANATION OF FIGURES

17 DPND activity in a lifer explant. Intense activity occurs in both parenchymal and connective tissue cells. $\times 600$.

18 DPND activity in CAM liver transplant. Activity is confined to the hepatic parenchymal cells. $\times 1,600$.

19-20 SDH activity in 5- and 15-day liver, respectively. SDII activity occurs in both parenchymal and connective tissue cells and is exclusively mitochondrial in localization. $\times 1,600$.

21 SDH activity in a liver explant. Only diffuse activity is present. $\times 1,600$.

22 GDH activity in 15-day liver. All activity appears to be mitochondrial in localization. $\times 800$. 

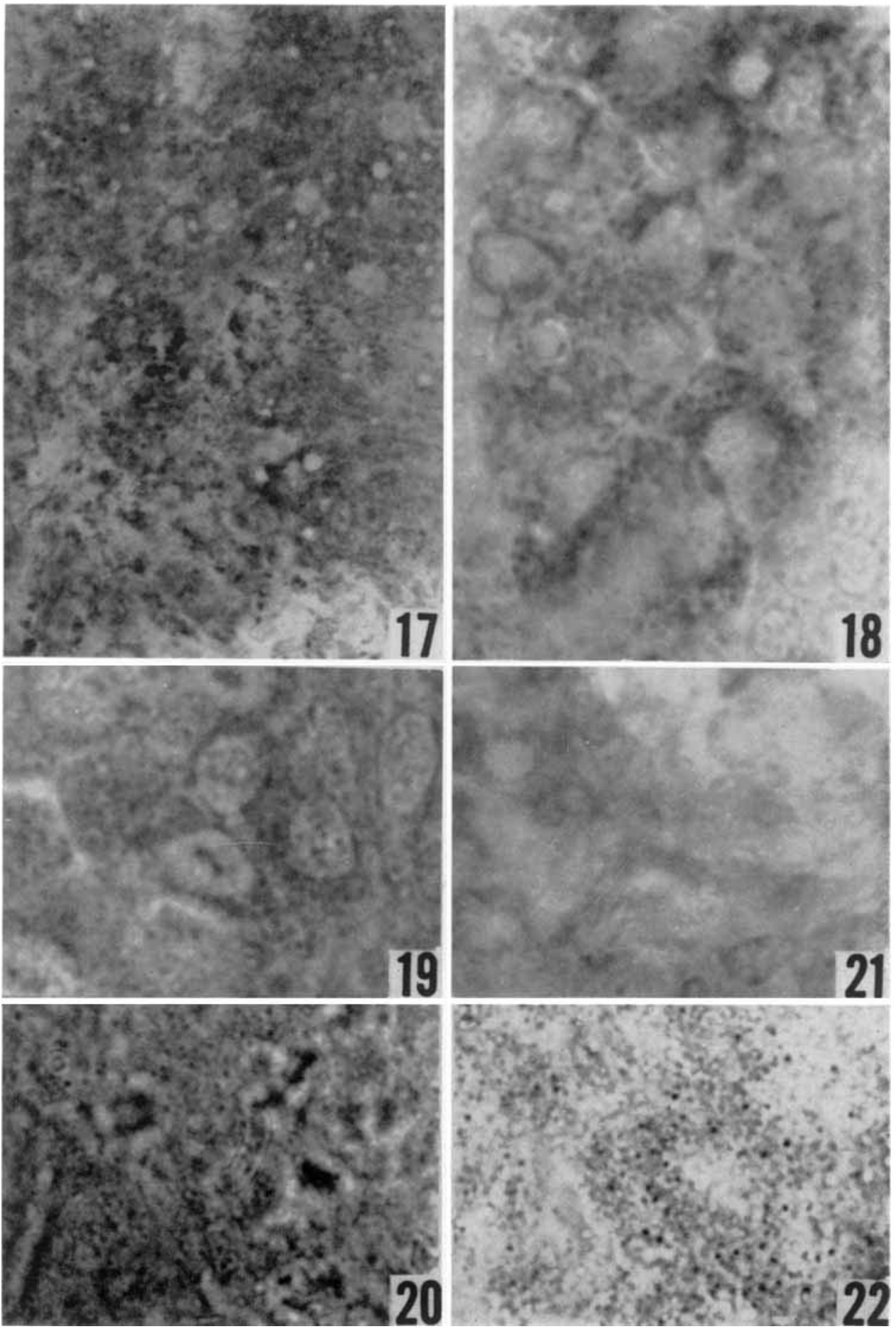
PLATE 6

EXPLANATION OF FIGURES

23 NSE activity in a five-day liver. Parenchymal cells contain moderate activity while connective tissue cells are weakly positive. $\times 400$.

24-25 NSE activity in 15-day liver. The parenchyma is strongly positive while little or no activity occurs in the connective tissue (arrow). $\times 400, \times 1,600$.

26 NSE activity in a CAM liver transplant. Activity appears as in the normal liver. $\times 1,600$. 

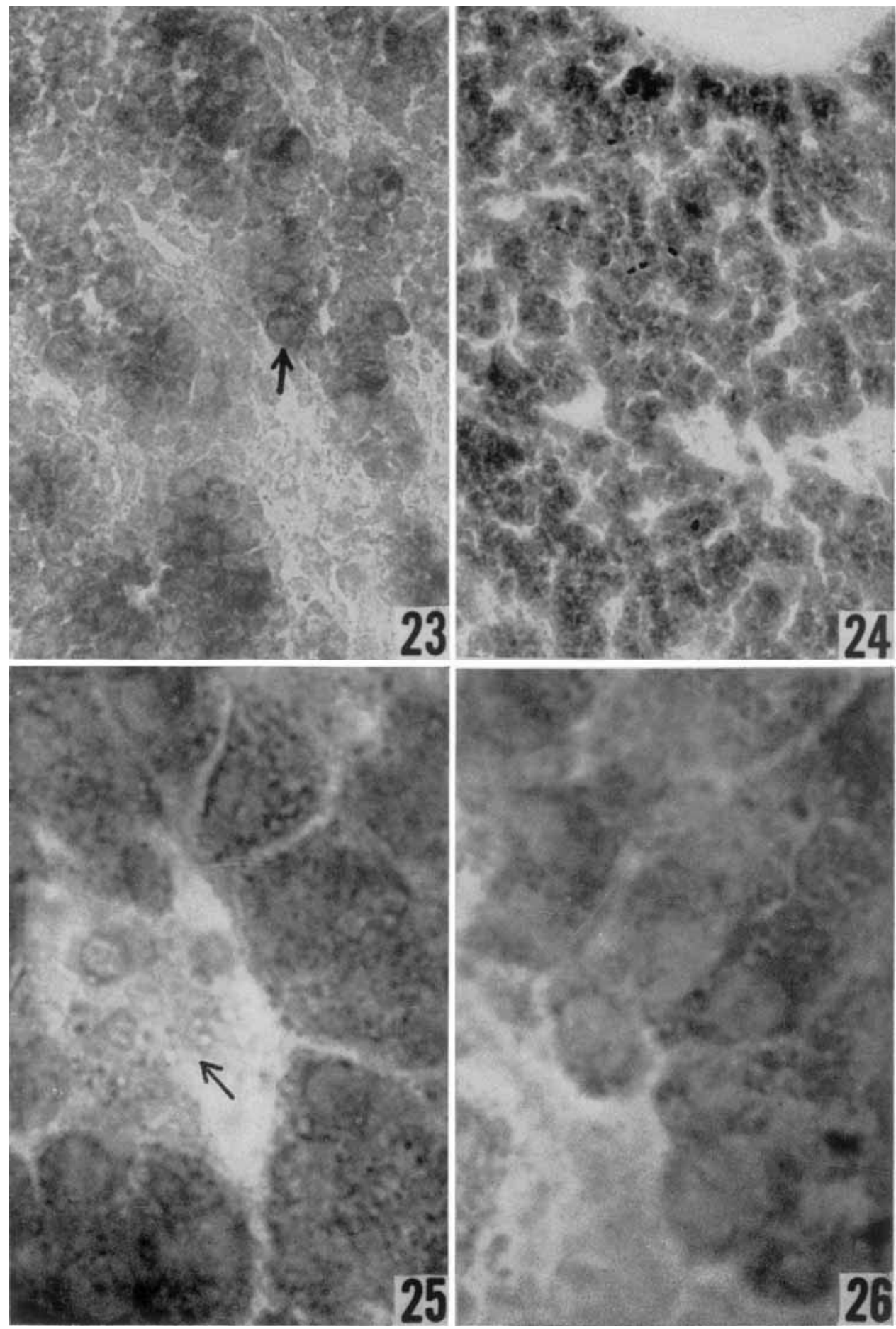